\title{
Single dose methotrexate for the treatment of unruptured ectopic pregnancy: experience in Kemaman Hospital, Terengganu, Malaysia
}

\author{
Azny Syahirah Mohamad Yusof*, Zahar Azuar Zakaria
}

Department of Obstetrics and Gynaecology, Hospital Kemaman, Jalan Da Omar, Kemaman, Terengganu, Malaysia

Received: 19 April 2016

Accepted: 13 May 2016

\section{*Correspondence:}

Dr. Azny Syahirah Mohamad Yusof,

E-mail: aznyd75@yahoo.com

Copyright: ( ) the author(s), publisher and licensee Medip Academy. This is an open-access article distributed under the terms of the Creative Commons Attribution Non-Commercial License, which permits unrestricted non-commercial use, distribution, and reproduction in any medium, provided the original work is properly cited.

\section{ABSTRACT}

Background: The objective of this study was to review our experience with single dose intramuscular methotrexate (MTX) for the treatment of ectopic pregnancy and to evaluate the safety and efficacy of the treatment modality.

Methods: A prospective study was carried out at Kemaman Hospital, Terengganu, Malaysia. A database was started and continued prospectively for patients meeting all the criteria's for MTX therapy from January 2015 till January 2016. The selection criteria's were patients who were asymptomatic or experience mild symptoms but haemodynamically stable and ectopic gestational mass of less than $4 \mathrm{~cm}$ on ultrasound. Intramuscular methotrexate (MTX) 50mg/m2 was administered as in-patient and monitored at least 24 hours for side effects. Upon discharge, blood samples were collected on day 4 and day 7 of MTX therapy and weekly to 2-weekly thereafter until hCG titers became $<2 \mathrm{mIU} / \mathrm{mL}$.

Results: 4 patients opted for medical treatment. All patients received only a single dose methotrexate and did not have complications post medical treatment. hCG level reached normalization within 30 days and 1 patient had longer follow up until 120 days. Higher patient satisfaction in all patients due to the noninvasive nature of the treatment modality.

Conclusions: This study showed that medical treatment of ectopic pregnancy with systemic single dose methotrexate can be a primary treatment modality for selected patients with stable unruptured tubal pregnancy.

Keywords: Methotrexate, Ectopic pregnancy

\section{INTRODUCTION}

Ectopic pregnancy is defined as implantation of fertilized ovum outside the uterine cavity. ${ }^{1}$ It is a medical emergency due to the high morbidity and mortality in the reproductive age group. ${ }^{2} 98 \%$ of ectopic pregnancies are in the fallopian tube, with a small percentage implanted at various sites such as ovaries, abdomen, and broad ligament. ${ }^{3}$

The exact aetiology is uncertain but several risk factors identified such as infertility, previous tubal surgery, contraceptive failure, previous ectopic pregnancy and smoking. ${ }^{4}$
Patients typically presented with lower abdominal pain and per vaginal bleeding in between 6th to 10th weeks of gestation. ${ }^{1}$ Haemodynamically unstable patients with syncope or signs of shock and positive urine pregnancy test should alert one to the diagnosis of ruptured ectopic pregnancy and should undergo surgery.

However in a stable patient, a less aggressive approach may be considered. While surgery is the mainstay of treatment, with the advent of early pregnancy ultrasound scan combined with quantitative b-HCG levels, this has facilitated the introduction of medical therapy with methotrexate (MTX) in the $1980 .^{5,6}$

The use of MTX to treat early unruptured ectopic pregnancy has been shown to be a safe and effective 
alternative treatment to surgery in properly selected cases. $^{7,8}$

In a standard practice in Kemaman Hospital, ectopic pregnancies were treated surgically. Patients with ruptured ectopic pregnancy and haemodynamically unstable will undergo emergency laparotomy, whilst those who are haemodynamically stable can be performed laparoscopically. Although surgery is definitive, it also carries a risk of anaesthetic and surgical related complications. In cases whereby patients had refused surgery or surgery may cause higher morbidity, options can be given to these patients. In Kemaman Hospital, a medical treatment by using methotrexate has been offered to these patients who were deemed fit to receive medical treatment according to the department's guidelines.

The purpose of this study is to review those patients with EP treated with MTX and to review the outcome.

\section{METHODS}

Patients referred to or admitted to gynaecological ward hospital Kemaman for suspected ectopic pregnancy or confirmed ectopic pregnancy from January 2015 till January 2016 was followed up. Patients who agreed or opted for medical treatment with methotrexate were followed up and included in this study. Patients who underwent surgical treatment or expectant management were excluded.

The following data were collected: demographic data including age, weight, height, presenting symptoms, methotrexate dose given, ultrasound findings including ectopic size, fluid in Pouch of Douglas, hCG level before, day 4 and day and of methotrexate administration. Data were analysed using SPSS software.

\section{RESULTS}

Table 1: Single dose methotrexate protocol for treatment of unruptured ectopic pregnancy.

\begin{tabular}{|ll|}
\hline Day & Therapy \\
0 & $\begin{array}{l}\text { hCG }(0 \text { and } 48 \mathrm{hrs}), \text { FBC, LFT, BUSE, } \\
\text { creatinine, ABO Rh }\end{array}$ \\
\hline 1 & IM MTX \\
\hline 4 & hCG \\
\hline 7 & hCG \\
\hline
\end{tabular}

Followed with weekly and 2-weekly; hCG=quantitative BhCG; $\mathrm{FBC}=$ full blood count; IM MTX = intramuscular methotrexate $50 \mathrm{mg} / \mathrm{m} 2$

The diagnoses were made by the ultrasound scan finding and serum BhCG levels. All patients received methotrexate intramuscularly based on body surface area (50mg X BSA). Basically all had received a dose of 50mg. Serum hCG level were taken on day 4 and day 7 after methotrexate injection, followed by weekly and 2 weekly review until serum level normalizes.

Table 2: Demographic and clinical data of 4 patients treated with single dose methotrexate.

\begin{tabular}{|l|l|}
\hline Characteristic & mean $+/-$ SD \\
\hline Age (years) & $28.75+/-2.98$ \\
\hline Parity & P0 to P2 \\
\hline Body weight $(\mathrm{kg})$ & $69.75+/-18.96$ \\
\hline Height $(\mathrm{cm})$ & $154+/-5.16$ \\
\hline BMI $(\mathrm{kg} / \mathrm{m} 2)$ & $29.25+/-9.28$ \\
\hline Pre-treatment hCG $(\mathrm{mIU} / \mathrm{ml})$ & 4788.5 \\
\hline Day 7 hCG & 1775 \\
\hline
\end{tabular}

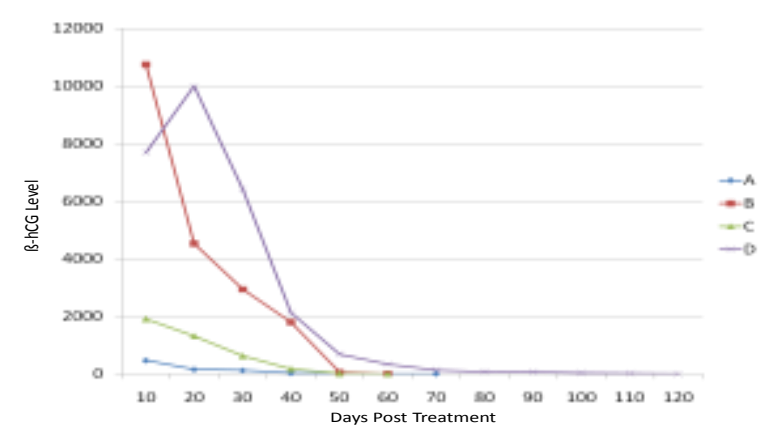

Figure 1: Plot of hCG titres (mIU/mL) against days post treatment in 4 representative patients treated with single dose methotrexate for unruptured ectopic pregnancy.

Patients' age was in between 25 to 32 years old. Before receiving methotrexate, all patients quantified their pain as mild. Size of ectopic pregnancy was in between 1.5 to $3.2 \mathrm{~cm}$ and only one patient revealed the presence of minimal intraperitoneal fluid collection. Serum BhCG levels were in the range of 388 to $10,743 \mathrm{mIU} / \mathrm{ml}$. 3 patients had normal serum hCG level after 30 to 33 days of injection of MTX. One patient took longer duration of 100 days. Basically all patients received single dose MTX. All patients did not complaint of any side effects such as gastritis, stomatitis or nausea.

There were 29 cases of ectopic pregnancies during the given time frame. 23 cases were treated surgically as primary mode of treatment. Out of 26 cases, 6 patients underwent emergency laparotomy and 17 patients underwent laparoscopic surgery. 2 cases opted for expectant management. 5 cases were suitable for medical treatment; however one case had to resort to emergency surgery due to ruptured tubal pregnancy and therefore had to be excluded from this review.

\section{DISCUSSION}

All patients diagnosed as unruptured tubal pregnancy will eventually undergo surgical treatment, either laparotomy or laparoscopy. However in recent years, there is a trend towards a nonsurgical approach. 
Medical treatment with methotrexate was first introduced as a successful treatment option for EP in the 1980's by Tanaka et al. ${ }^{9}$ Many literatures give different success rate of medical management in resolving an EP. A study conducted in Saudi Arabia revealed a 72\% success rate, whilst study in Scandinavia and Makkah showed a success rate of $78 \%$ and $100 \%$ respectively. ${ }^{10-12}$

In our experience in Kemaman Hospital, this review demonstrates that selective patients with an unruptured ectopic pregnancy can be safely and effectively treated with single IM dose of methotrexate in the manner described. No patients in this study experienced any methotrexate side effects such as gastritis, nausea, and stomatitis. Serum hCG level normalized within 30 days of injection in 3 cases, whilst one patient need longer follow up until 120 days to reach a normal hCG level. Fortunately, all patients were successfully treated with only a single dose injection. This regimen is more attractive than multiple doses as it virtually eliminates the incidence of chemotherapeutic side effects as less methotrexate is required, minimizes hospitalization, reduced number of follow up and less costly.

By confirming the diagnosis of ectopic pregnancy in early gestation by ultrasound scan, hence avoiding the need for laparoscopy in this diagnosis, this can reduced the potential cost and morbidity of hospitalization and surgery. Patients who have been successfully treated with this method may return to their daily routine activities immediately, hence can avoid burdens associated with lost wages or child care concern. One patient had ruptured ectopic pregnancy prior to medical treatment; in this case the size of the tubal mass was slightly larger $(4 X 3.5 \mathrm{~cm})$ and had minimal intraperitoneal blood collection. None of the ectopic had cardiac activity.

By having a higher threshold for laparoscopy and reasserting the safety of methotrexate, this review provides an alternative modality for the treatment of ectopic pregnancy. The opponents of medical treatment will point to the need for regular and long follow up, with possible loss to follow up, however this has to be balanced against the risk and complications of surgery involved and future fertility. Even in a conservative surgery such as salpingostomy, a similar follow up is put to the patient similar to the primary medical treatment. Because of the non-invasive nature of MTX treatment, it is more favourable to the patients and this translates into a higher patients' satisfaction as they can avoid surgery and longer hospital stay, they can return to work early and avoid unnecessary child care concern.

Surgery may no longer be a primary treatment for the diagnosis of unruptured ectopic pregnancy as evidence show that alternative medical treatment is safe, effective and can preserve the fallopian tube in those who still desire for future fertility.

\section{CONCLUSION}

In conclusion, medical treatment can be offered as a primary treatment for a case of stable, unruptured ectopic pregnancy in tandem with the hospital's guidelines and protocols.

\section{Funding: No funding sources}

Conflict of interest: None declared

Ethical approval: The study was approved by the Institutional Ethics Committee

\section{REFERENCES}

1. Walker JJ. Ectopic pregnancy. Clinical Obstetrics and Gynaecology. 2007;50(1):89-99.

2. Della-Guistina D, Denny M. Ectopic Pregnancy. Emergency Medicine Clinics of North America. 2003;21(3):565-84.

3. Varma R, Gupta J. Tubal ectopic pregnancy. Clinical Evidence. 2009:1406.

4. Sivalingam V, Duncan W, Kirk E, Shephard L. Diagnosis and management of ectopic pregnancy. Journal of Family Planning and Reproductive Health Care. 2011;37:231-40.

5. Rodi IA, Sauer MV, Gorril MJ. The medical treatment of unruptured ectopic pregnancy with methotrexate and citrovorum rescue:preliminary experience. Fertility and Sterility. 1986;46(5):811-3.

6. Stovall TG, Ling FW, Buster JE. Outpatient chemotherapy of unruptured ectopic pregnancy. Fertility and Sterility. 1989;51(3):435-8.

7. Stovall TG, Ling FW. Single dose methotrexate: an expanded clinical trial. The American Journal of Obstetrics and Gynaecology. 1993;168(6):1759-65.

8. Hajenius PJ, Engelsbel S, Mol BWJ. Randomised trial of systemic methotrexate versus laparoscopic salpingostomy in tubal pregnancy. The Lancet. 1997;350(9080):774-9.

9. Morlock RJ, Lafata JE, Eisenstein D. Costeffectiveness of single dose methotrexate compared with laparoscopic treatment of ectopic pregnancy. Obstetrics and Gynaecology. 2000;95(3):407-12.

10. Sendy F, Alshehri E, Alajmi A. Failure rate of single dose methotrexate in management of ectpic pregnancy. Obstetrics and Gynaecology International. 2015 Article ID 902426, 5 pages.

11. Potter MB, Lepine LA, Jamieson DJ. Predictors of success with methotrexate treatment of tubal ectopic pregnancy at Grady Memorial Hospital. The American Journal of Obstetrics and Gynaecology. 2003;188(5):1192-4.

Cite this article as: Mohamad Yusof AS, Zakaria ZA. Single dose methotrexate for the treatment of unruptured ectopic pregnancy: experience in Kemaman Hospital, Terengganu, Malaysia. Int J Reprod Contracept Obstet Gynecol 2016;5:1754-6. 